\title{
Effective Usage of Available Spectrum Using Dispersion Detection Technique
}

\author{
Durga $\mathrm{R}^{\mathrm{a}}$ and Selvaraj $\mathrm{D}^{\mathrm{b}}$ \\ ${ }^{a} P G$ Scholar, Detof ECE, Panimalar Engineering College, Chennai \\ ${ }^{\mathrm{b}}$ Professor, Deptof ECE, Panimalar Engineering College, Chennai
}

\begin{abstract}
Spectrum sensing techniques are used for aquisingthe frequency spectrum in cognitive radio. From research, the efficiency of the spectrum sensing technique increases only if its complexity is increased and if its complexity is decreased then its efficiency decreases. so, a new technique is proposed in this paper based on Dispersion Detection (DD) to balance both complexity and efficiency. Using this detection technique, the false alarm probability is derived for multiple antenna using test statistic distribution. The decision threshold is derived to provide the accurate results. The derived values are verified with Monto Carlo simulation.
\end{abstract}

Keywords. Spectrum sensing, dispersion detection, false alarm, decision threshold

\section{Introduction}

Cognitive radio is defined as a upgrading technology for easing the spectrum shortage problem by utilizing the licensed band. There are various types of sensing techniques such as energy technique, matched filter technique, cyclostationary technique, Loglikelihood Ratio Test (LRT) techniques which contain both advantages and disadvantages. Some detection technique requires channel information and some technique doesn't require channel information. The spectrum sensing using multiple antennas can improve the transmission rate and it can also increase the capacity of the channel. In [10] the multiple antenna case exploit covariance and achieve low $P_{f}$ detection rate by increasing the number of samples. The covariance detection method [9] provides the best signal strength from $-6 \mathrm{db}$ the detection performance is increased with an enhanced SNR. In [8] the detection performance based on the noise signal is achieved at $-10 \mathrm{db}$ even in the existence of a noise signal. [7] the aim of this method is to separate the signal from its background noise. this method provides better results than energy detection by calculating different parameters for different number of antennas. [6] LRT is an optimal method but its quite complex and it requires channel information beforehand.[5]

\footnotetext{
${ }^{1}$ Durga R PG Scholar, Department of ECE, Panimalar Engineering College, India Email:durga.1997r@gmail.com
} 
The signal space analysis methodology is analyzed by capturing the data information from RF platform to analyze the robustness and to reduce the framework issues by providing better performance. [4] the analysis of test statistic id derived under signal absence hypothesis moment matching method is exploited to derive the distribution of gamma and by this, it provides better analysis for the given threshold.[1] the generalized order statistics and OR rule using a data fusion algorithm is analyzed to initialize protection due to non-homogenous signals. [2] the throughput maximization analysis is analyzed using local sensing optimization and fusion optimization. [3]The detection performance is not enhanced even when the samples are 50000.

\section{Dispersion detection method}

Dispersion is a measure that gives an idea about the scattering of the values. Some of the different measures of dispersion are Range, Mean, correlation, Variance and Coefficient of variance.

\subsection{The Signal Model}

Dispersion detection can differentiate the power and noise of the signal which is a drawback of interference feature detection and energy detection. The proposed technique is used for avoiding the uncertainty of noise power. The dispersion detection attempts on 1) noise samples are not dependent and hence not correlated 2) samples of reasonable information-carrying signals are correlated.Consider $M$ antennas utilized. be the discrete-time sample received signal form the $\mathrm{M}^{\text {th }}$ antenna. The statistical dispersion matrix of the discrete-time sample obtained signal with binary hypotheses are defined as (Eq. 1)

$$
x(k)=\left\{\begin{array}{l}
n(k), H_{0} \\
s(k)+n(k), H_{1}
\end{array}\right.
$$

i.e., the obtained signal $\mathrm{x}(\mathrm{k})=\left[\mathrm{x}_{1}(\mathrm{k}), \mathrm{x}_{2}(\mathrm{k}), \ldots . \mathrm{x}_{\mathrm{n}}(\mathrm{k})\right]$ consists only of Independent and identically dessimated (IID) with zero mean additive white gaussian noise (AWGN)

$$
\begin{array}{r}
\mathrm{n}(\mathrm{k})=\left[\mathrm{n}_{1}(\mathrm{k}), \mathrm{n}_{2}(\mathrm{k}) \ldots \ldots \mathrm{n}_{\mathrm{M}}(\mathrm{k})\right] \\
n(k)=N\left(0, \sigma_{n}^{2} I_{M}\right)
\end{array}
$$

The dispersion detection of obtained signal is given by

$$
R_{x}=E\left[x(k) x^{T}(k)\right]
$$

Then,

$$
R_{x}=\left\{\begin{array}{l}
\sigma^{2}{ }_{n} I_{M}, H_{o} \\
R s+\sigma^{2}{ }_{n} I_{M}, H_{1}
\end{array}\right.
$$

Then, $\mathrm{x}(\mathrm{k})$ is a multivariate random vector with general properties

$$
x(k) \approx\left\{\begin{array}{l}
N\left(0, \sigma^{2}{ }_{n} I_{M}\right), H_{0} \\
N\left(0, R_{s} \sigma^{2}{ }_{n} I_{M}\right), H_{1}
\end{array}\right.
$$

If the PU signal is not present $R_{x}$ then the slanting values are zeros. If the PU signal is exists then the non-diagonal elements should be related to signal and power which should not be affected by correlation.

$$
T=\frac{T_{1}}{T_{2}}
$$


$T_{1}$ represents non-diagonal elements and $T_{2}$ represents diagonal elements i.e. power signal where, and

$$
T_{2}=\sum_{p=1}^{M}\left|R_{x}(p, p)\right|
$$

\subsection{Statistical Dispersion Detection}

The sample dispersion estimates $\mathrm{R}_{\mathrm{x}}$ are calculated based on the obtained received signal (Eqs8 to 16)

$$
\hat{R}_{x}=\frac{1}{k} \sum_{k=1}^{k} x(k) x^{T}(k)
$$

The test dispersion is given by

$$
\widehat{T}=\frac{T_{1}}{T_{2}}
$$

Where,

$$
\widehat{T}_{1}=\sum_{p \neq q ; p, q=1}^{M}\left|R_{x}(p, q)\right|
$$

and

$$
\widehat{T}_{2}=\sum_{p=1}^{M}\left|R_{x}(p, p)\right|
$$

The decision threshold is given by

$$
\text { decision }=\left\{\begin{array}{l}
H_{0}, \widehat{T}<\lambda \\
H_{1}, \widehat{T}>\lambda
\end{array}\right.
$$

The $\mathrm{P}_{\mathrm{d}}$ and $\mathrm{P}_{\mathrm{f}}$ is given by

$$
\begin{aligned}
& P_{f}=P_{r}\left(\widehat{T}>\lambda \mid H_{0}\right) \\
& P_{d}=P_{r}\left(\hat{T}>\lambda \mid H_{1}\right)
\end{aligned}
$$

The $P_{d}$ and $P_{f}$ is given by

$$
\begin{aligned}
& P_{d}=1-F_{\widehat{T} \mid H_{1}}(\lambda) \\
& P_{f}=1-F_{\widehat{T} \mid H_{0}}(\lambda)
\end{aligned}
$$

\subsection{The Decision Threshold}

For a best detection technique, a high $\mathrm{P}_{\mathrm{d}}$ and low $\mathrm{P}_{\mathrm{f}}$ should be obtained. The threshold isundermined between $P_{d}$ and $P_{f}$. The threshold is chosen such that a certain value of false alarm probability $\mathrm{P}_{\mathrm{f}}$ is achieved. The threshold selection can be based on either theoretical derivation or computer simulation. 


\subsubsection{Threshold selection based on computer simulation}

Initially, $\mathrm{P}_{\mathrm{f}}$ value is set and a threshold ' $\mathrm{l}$ ' is found to meet the required $\mathrm{P}_{\mathrm{f}}$. An AWGN is generated as the input signal and the threshold is adjusted to meet the required $\mathrm{P}_{\mathrm{f}}$.

\section{Results}

\section{$3.1 \boldsymbol{P}_{f}$ versus $S N R$}

Fig. 1 shows the $P_{f}$ versus SNR for a fixed number of antennas and different k(number of samples) and in fig. 2 the number of antennas is reduced. By reducing the number of antennas the result shows is for high SNR the $P_{f}$ is low and for low SNR the $P_{f}$ is high. By increasing the SNR values the false alarm probability can be reduced using dispersion detection.
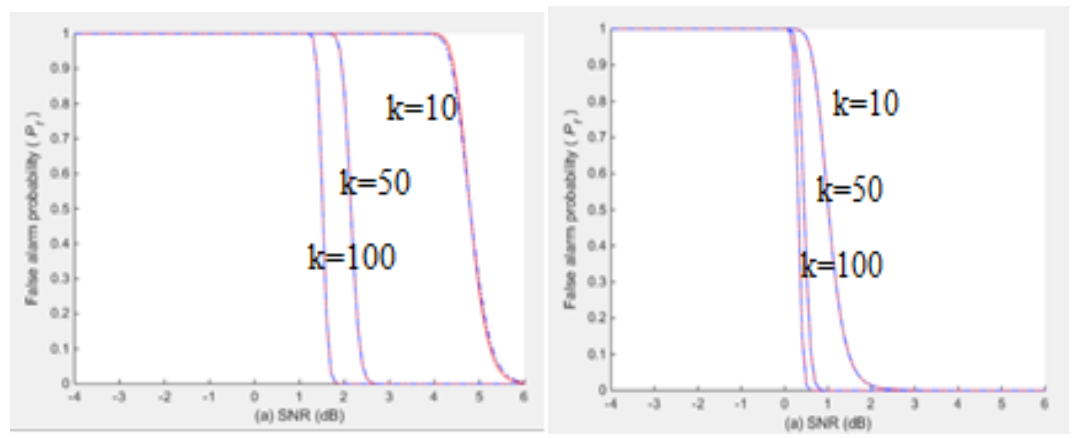

Figure 1. $P_{\mathrm{f}}$ versus $\mathrm{SNR}$ for $\mathrm{k}=10,50,100$ (a) $\mathrm{M}=20$ (b) $\mathrm{M}=5$
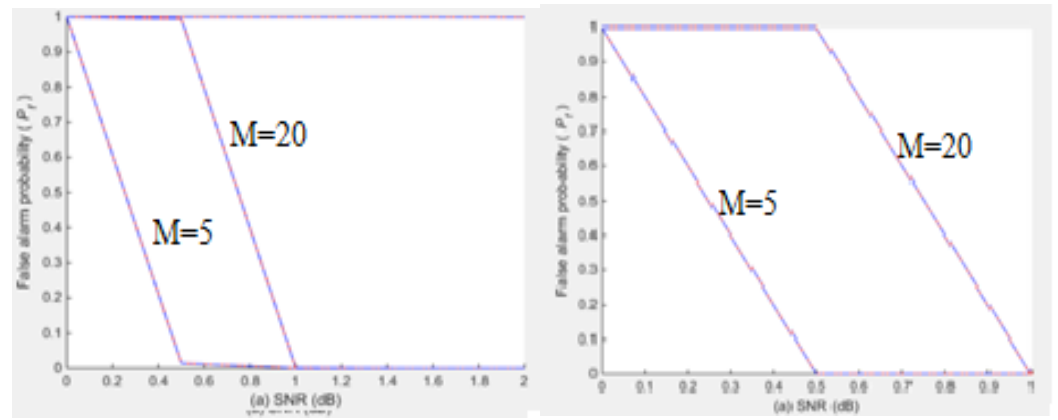

Figure 2. $P_{f}$ versus $S N R$ for $M=5,20$ (a)k=100 (b) $k=1000$

\section{2. $P_{f}$ versus $\lambda$}

The $P_{f}$ versus threshold is calculated as fixed number of antennas with that of different samples is shown in the figure.for different ranges of $\mathrm{k}$ is simulated.The results are given for fixed $\mathrm{M}=5$ in fig. and $\mathrm{M}=20$ in fig.when the signal power and noise power is above the threshold there will be low false alarm probability. 


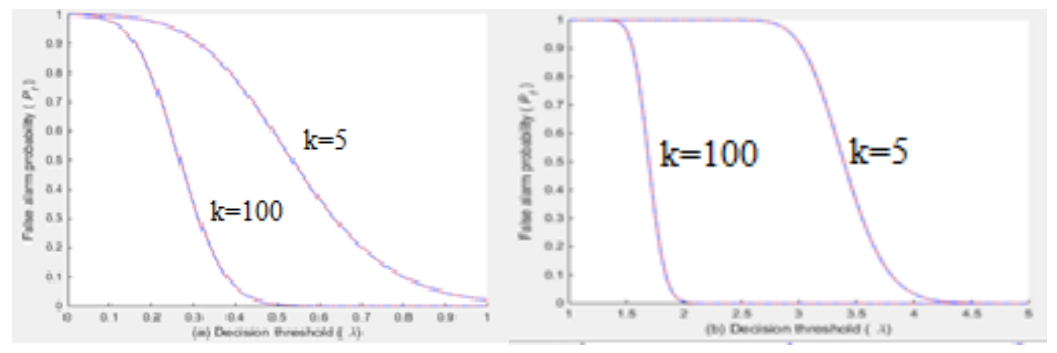

Figure 3. $P_{f}$ versus $\lambda$ for $k=5,100$ (a) $M=5$ (b) $M=20$

\section{Conclusion}

In this paper, the sensing algorithm of the dispersion detection is proposed. By receiving the number of samples, the received signal is exploited. The test statistics are provided for setting the threshold and obtain the probability of false alarm. Dispersion detection is doesn't require any signal information beforehand which is an advantage. The false alarm probability vs. SNR is calculated. This detection technique provides a low false alarm probability even under high SNR with the presence of noise.

\section{References}

[1] Madan H T. P I Basarkod. An Optimized and Trained Model of Cooperative Sensing for Cognitive Radio Networks. International Journal of Recent Technology and Engineering (IJRTE) Volume-8 Issue3, September 2019.

[2] Chang-Joo Kim. EunSook Jin. Kyung-yul Cheon. Seon-Hwan Kim. Robust spectrum sensing under noise uncertainty for spectrum sharing. ETRI journal, volume 41, Issue 2, pp.176-183 2018.

[3] Y. Zeng, and Y. C. Liang, Spectrum sensing algorithms for cognitive radio based on statistical covariance," IEEE Trans. Veh. Technol., vol. 58, no. 4, pp. 1804-1815, May 2009.

[4] Lei Huang. Cheng Qian. Yuhang Xiao. Q. T. Zhang. Performance Analysis of Volume-Based Spectrum Sensing for Cognitive Radio IEEE Transactions On Wireless Communication. IEEE volume 14, Issue 1. 2015.

[5] Q.T. Zhang. Advanced Detection Techniques for Cognitive Radio. IEEE 2009

[6] Shunchao Zhang, Yonghua Wang. Hantao Yuan. Pin Wan. Yongwei Zhang. Multiple-Antenna Cooperative Spectrum Sensing Based On The Wavelet Transform And Gaussian Mixture Model. NCBI. Sensor(Basel) Volume 18, Issue 1, 2019.

[7] Vishal Sharma, Richa Sharma Analysis Of Spread Spectrum In Matlab International Journal of Scientific \& Engineering Research, Volume 5, Issue 1, January-2014

[8] K.Nayomi. M.Sailaja. Novel Spectrum-Sensing Algorithms for Cognitive Radio Based on with and without Signal Information. International Journal for Modern Trends in Science and Technology Volume: 03, Issue No: 01, January 2017.

[9] Vivek Kumar Baghel. Khan. Covariance Based Spectrum Detection for Cognitive Radio. International Journal of Science and Research (IJSR), Issue 10th April, pp.391 to 394, 2015.

[10] YonghongZeng. Ying-Chang Liang. Spectrum-Sensing Algorithms for Cognitive Radio Based on Statistical covariances. IEEE transactions on vehicular technology, vol. 58, no. 4, may 2009 